\title{
SMb20651 is another acyl carrier protein from Sinorhizobium meliloti
}

Correspondence

Isabel M. López-Lara

isabel@ccg.unam.mx

Received 9 July 2008

Revised 19 September 2008

Accepted 2 October 2008

\author{
Ana Laura Ramos-Vega, ${ }^{1}$ Yadira Dávila-Martínez, ${ }^{1}$ Christian Sohlenkamp, ${ }^{1}$ \\ Sandra Contreras-Martínez, ${ }^{2}$ Sergio Encarnación, ${ }^{2}$ Otto Geiger ${ }^{1}$ \\ and Isabel M. López-Lara ${ }^{1}$
}

${ }^{1}$ Programa de Ecología Genómica, Centro de Ciencias Genómicas, Universidad Nacional Autónoma de México, Apdo. Postal 565-A, Cuernavaca, Morelos C. P. 62251, Mexico

${ }^{2}$ Programa de Genómica Funcional de Procariontes, Centro de Ciencias Genómicas, Universidad Nacional Autónoma de México, Apdo. Postal 565-A, Cuernavaca, Morelos C. P. 62251, Mexico

Acyl carrier proteins (ACPs) are small acidic proteins that carry growing acyl chains during fatty acid or polyketide synthesis. In rhizobia, there are four different and well-characterized ACPs: AcpP, NodF, AcpXL and RkpF. The genome sequence of Sinorhizobium meliloti 1021 reveals two additional ORFs that possibly encode additional ACPs. One of these, smb20651, is located on the plasmid pSymB as part of an operon. The genes of the operon encode a putative asparagine synthetase (AsnB), the predicted ACP (SMb20651), a putative long-chain fatty acylCoA ligase (SMb20650) and a putative ammonium-dependent $N A D^{+}$synthetase (NadE1). When $\mathrm{SMb} 20651$ was overexpressed in Escherichia coli, $\left[{ }^{3} \mathrm{H}\right] \beta$-alanine, a biosynthetic building block of $4^{\prime}$-phosphopantetheine, was incorporated into the protein in vivo. The purified SMb20651 was modified with 4'-phosphopantetheine in the presence of S. meliloti holo-ACP synthase (AcpS). Also, holo-SMb20651 was modified in vitro with a malonyl group by malonyl CoA-ACP transacylase. In E. coli, coexpression of SMb20651 together with other proteins such as AcpS and SMb20650 led to the formation of additional forms of SMb20651. In this bacterium, acylation of $\mathrm{SMb} 20651$ with $\mathrm{C} 12: 0$ or $\mathrm{C} 18: 0$ fatty acids was detected, demonstrating that this protein is involved in fatty acid biosynthesis or transfer. Expression of SMb20651 was detected in $S$. meliloti as holo-SMb20651 and acyl-SMb20651.

\section{INTRODUCTION}

Acyl carrier proteins (ACPs) are small acidic proteins that are key cofactors in fatty acid and polyketide syntheses. Acyl intermediates are covalently bound to an ACP, which shuttles them from one enzyme to another. The linkage between fatty acyl groups and ACP is achieved by a thioester bond in which the thiol of the ACP $4^{\prime}$ phosphopantetheine prosthetic group is condensed with the carboxy group of fatty acids. Apo-ACP is converted to the active protein by holo-ACP synthase (AcpS), which transfers the $4^{\prime}$-phosphopantetheine from coenzyme A (CoA) to apo-ACP (Flugel et al., 2000; Lambalot et al., 1996), thereby converting it to the functional holo-ACP.

Abbreviations: ACP, acyl carrier protein; AcpS, holo-ACP synthase; CTAB, cetyltrimethylammoniumbromide; DOC, sodium deoxycholate.

Two supplementary figures, showing results of RT-PCR analysis demonstrating that $a s n B, s m b 20651, s m b 20650$ and nadE1 are encoded in an operon, the scheme of asnB, smb20651, smb20650 and nadE1 arrangement, and a multiple sequence alignment of the $S$. meliloti acyl carrier proteins and selected SMb20651 homologues, are available with the online version of this paper.
In Escherichia coli $\mathrm{K}-12$, the only ACP is AcpP. This essential and constitutive protein is involved not only in the synthesis of fatty acyl chains but also in their transfer during phospholipid, lipid A or haemolysin synthesis (reviewed by Byers \& Gong, 2007). Acyl-ACP also functions as acyl donor during the synthesis of $N$ acylhomoserine lactones in Gram-negative bacteria (Fuqua \& Greenberg, 2002) and of $\gamma$-butyrolactones in Streptomyces (Kato et al., 2007). Bacteria with complex metabolism often have specialized ACPs and ACP homologues that are involved in the synthesis of secondary metabolites such as polyketides or non-ribosomal peptides (Geiger \& López-Lara, 2002; Lai et al., 2006), or in the synthesis of D-alanyl-lipoteichoic acid (Neuhaus \& Baddiley, 2003).

Rhizobia have four well-characterized ACPs (Geiger \& López-Lara, 2002): AcpP for general fatty acid synthesis (Platt et al., 1990; López-Lara \& Geiger, 2000), NodF for the synthesis of polyunsaturated fatty acids and for their transfer to nodulation factors (Ritsema et al., 1998), AcpXL for the synthesis and transfer of the 27-hydroxyoctacosanoic acid during rhizobial lipid A synthesis (Brozek et al., 
1996), and RkpF, which participates in the synthesis of rhizobial capsular polysaccharides (Epple et al., 1998). The genome sequence of Sinorhizobium meliloti 1021 (Galibert et al., 2001) reveals the existence of two additional putative ACPs (Geiger \& López-Lara, 2002). One of these is encoded by the gene smb20651 and is located in the plasmid pSymB, forming part of a possible operon of four genes (Fig. 1). The SMb20651 protein was assigned in the genome project as a hypothetical protein. However, it has all the characteristics of an ACP. SMb20651 is small (88 aa) and acidic (isoelectric point 3.8), and has a predicted $4^{\prime}$ phosphopantetheine binding motif. The genes of the operon code for a putative asparagine synthetase (AsnB), the predicted ACP (SMb20651), a putative long-chain (C12-C18) fatty acyl CoA ligase (SMb20650) and a putative ammonium-dependent $\mathrm{NAD}^{+}$synthetase (NadE1).

Here, we present the characterization of the protein SMb20651 as an ACP; SMb20651 incorporates the 4'phosphopantetheine prosthetic group, can be modified with a malonyl group and can carry $\mathrm{C} 12: 0$ and $\mathrm{C} 18: 0$ fatty acyl chains. Although no phenotype was associated with the absence of SMb20651 in S. meliloti, different modified forms of SMb20651 could be detected in the wild-type strain, indicating that the protein is actively participating in an acyl-involving metabolic pathway.

\section{METHODS}

Bacterial strains, plasmids and growth conditions. Bacterial strains and plasmids used in this work and their relevant characteristics are listed in Table 1. E. coli strains were grown at $30{ }^{\circ} \mathrm{C}$ either in Luria-Bertani (LB) broth (Sambrook \& Russell, 2001) or in M9 minimal broth (Miller, 1972), for $\left[{ }^{3} \mathrm{H}\right] \beta$-alanine labelling assays. S. meliloti strains were grown at $30{ }^{\circ} \mathrm{C}$ either in complex tryptone yeast (TY) broth supplemented with $4.5 \mathrm{mM} \mathrm{CaCl}{ }_{2}$ (Beringer, 1974) or in one of the following minimal media: M9 supplemented with $2 \mu \mathrm{g}$ biotin $\mathrm{ml}^{-1}$ and succinate $(10 \mathrm{mM})$ replacing glucose as the carbon source, Sherwood (1970) medium with succinate $(8.3 \mathrm{mM})$ replacing mannitol as the carbon source, or Robertsen et al. (1981) medium. In experiments designed to evaluate the influence of the nitrogen source on growth, glutamate in Robertsen medium was replaced by $\mathrm{NH}_{4} \mathrm{Cl}$. In other experiments, glutamate and succinate were replaced by glutamine or asparagine as carbon and nitrogen sources, respectively. To study salt tolerance, cultures were grown in complex TY medium supplemented with 0.1 , $0.25,0.5$ or $0.7 \mathrm{M} \mathrm{NaCl}$. Antibiotics were added, when required, to the following final concentrations $\left(\mu \mathrm{g} \mathrm{ml} \mathrm{m}^{-1}\right)$ : carbenicillin, 100; chloramphenicol, 20; kanamycin, 50; gentamicin, 20; spectinomycin, 200; and tetracycline, 20 for E. coli; and neomycin, 200; spectinomycin, 400; streptomycin, 500; and tetracycline, 4 for S. meliloti.

\section{$0.5 \mathbf{~ k b}$}

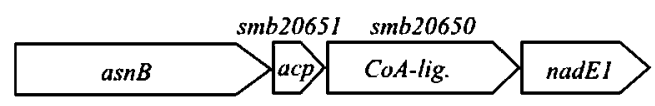

\section{Sinorhizobium meliloti 1021}

Sinorhizobium medicae WSM 419

Mesorhizobium sp. BNC1

Planctomyces maris DSM 8797

Rhodopseudomonas palustris Bis $\mathrm{A} 53$

Roseiflexus castenholzii DSM 13941

Geobacter bemidjiensis

Anaeromyxobacter dehalogenans 2CP-C

Mesorhizobium huakuii MAFF303099

Sorangium cellulosum 'So Ce 56'

Azoarcus sp. EbN1

Methanosarcina barkeri

Agrobacterium tumefaciens C58

Fig. 1. Genetic organization around the gene encoding SMb20651 and around selected SMb20651 homologues in different organisms. Nomenclature used for comparison: acp, acyl carrier protein homologous to SMb20651; CoA-lig., long-chain CoAligase homologous to SMb20650; asnB, putative asparagine synthetase; nadE1, putative NAD synthetase; ppt, phosphopantetheinyl transferase. 
Table 1. Bacterial strains and plasmids used in this study

$\mathrm{Kn}^{\mathrm{R}}, \mathrm{Cb}^{\mathrm{R}}, \mathrm{Cm}^{\mathrm{R}}, \mathrm{Gm}^{\mathrm{R}}, \mathrm{Spc}^{\mathrm{R}}, \mathrm{Tc}^{\mathrm{R}}$, kanamycin, carbenicillin, chloramphenicol, gentamicin, spectinomycin and tetracycline resistance, respectively. Sm and Ec denote genes from S. meliloti 1021 and E. coli, respectively. Plasmids pAL03 to pAL06 are described in Methods, Construction of smb20651 mutants.

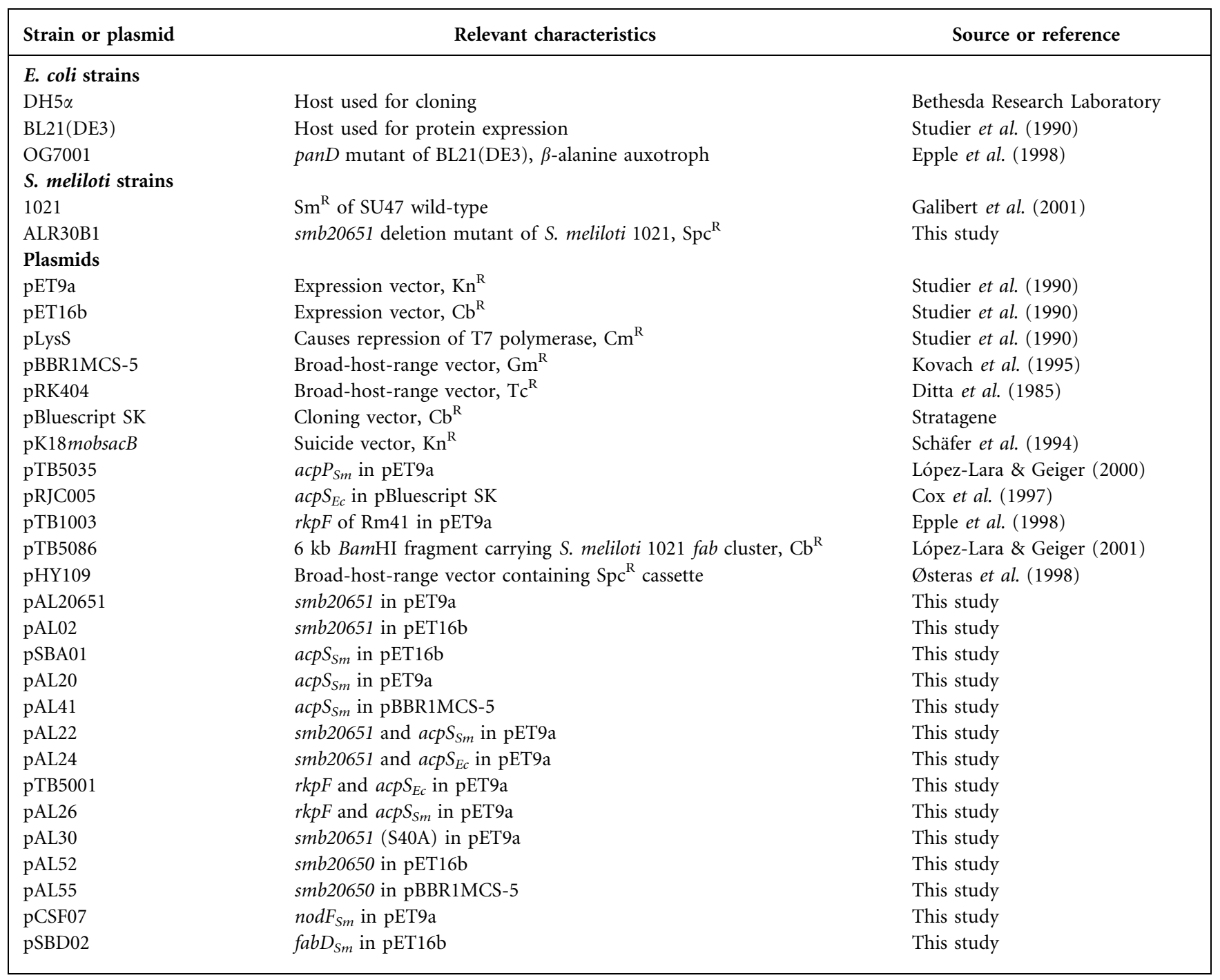

Plasmids pRK404, pBBR1MCS-5 and pK18mobsacB and their derivatives were mobilized into $S$. meliloti strains by triparental mating using the mobilizing plasmid pRK2013, as described elsewhere (Figurski \& Helsinki, 1979).

DNA manipulations and RT-PCR analysis. Recombinant DNA techniques were carried out using standard procedures (Sambrook \& Russell, 2001). Commercial sequencing of amplified genes by Eurofins Medigenomix (Martinsried, Germany) corroborated the correct DNA sequence. DNA and derived protein sequences were analysed using the National Center for Biotechnology Information (NCBI) BLAST network centre (Altschul et al., 1997). RNA was isolated from $2 \mathrm{ml}$ of culture using TRIzol reagent (Invitrogen) and treated with DNase I (Fermentas) as indicated by the manufacturers. cDNA was synthesized using a RevertAid H Minus First Strand cDNA synthesis kit (Fermentas) as specified by the manufacturer.

Construction of expression plasmids. All genes were amplified from S. meliloti 1021 genomic DNA, with the exception of smc00571 ( $f a b D)$, which was amplified from plasmid pTB5086 (López-Lara \& Geiger, 2001). Using specific oligonucleotides (Table 2), the genes smb20651, smb20650, fabD, nodF (sma0852) and acpS (smc02654) were amplified and, after restriction with $\mathrm{NdeI}$ and BamHI (or NdeI and BglII in the case of smb20650), the fragments were cloned into pET9a and/or pET16b (Studier et al., 1990) that had been digested with NdeI and BamHI. Names given to the respective expression plasmids are given in Table 1. Plasmid pAL20 was digested with BamHI and BglII to reclone the acpS gene region into the BamHI-digested broad-host-range vector pBBR1MCS-5 (Kovach et al., 1995) to yield plasmid pAL41.

For coexpression of SMb20651 with AcpS from S. meliloti, plasmid pAL20 was digested with BamHI and BgIII and the 420 bp fragment carrying S. meliloti acpS was cloned into plasmid pAL20651 that had been digested with BamHI, to yield pAL22. Similarly, the E. coli acpS gene $\left(a c p s_{E c}\right.$ ) was obtained after digesting the plasmid pRJC005 (Cox et al., 1997) with BglII and then cloning the fragment into BamHIdigested pAL20651 to yield pAL24. Plasmids for coexpression of the protein RkpF from S. meliloti Rm41 with S. meliloti AcpS or E. coli 
Table 2. Oligonucleotide primers used in this study

For construction of expression plasmids, the forward primers incorporated an NdeI restriction site (underlined) overlapping the start codon of the respective genes. The reverse primers incorporated a BamHI (or BglII in the case of smb20650) restriction site (underlined) after the stop codon.

\begin{tabular}{|ll|}
\hline Primer & \multicolumn{1}{c|}{ sequence $\mathbf{( 5}^{\prime} \mathbf{- 3}^{\prime} \mathbf{)}$} \\
\hline $\begin{array}{l}\text { Primers used for construction of expression plasmids } \\
\text { smb20651F }\end{array}$ & \\
smb20651R & AGGAATACATATGACCCAAGCGATTAAGGAC \\
acpSF & AAAGGATCCTTACGCCGAGACCGGCACG \\
acpSR & AGGAATACATATGATCATCGGTATCGGCAG \\
smb20650F & AAGGATCCCTAAACCGTTCCTCGGC \\
smb20650R & AGGAATACATATGCGGTTCGAGCAATTCCTC \\
fabDF & AAATAAGATCTCATTCTGCGGGCTCCAATG \\
fabDR & AGGAATACATATGAGCATCGCATTCACGTTCC \\
nodFF & AAAGGATCCTCAGGCCAAGAGGACGGCAAG \\
nodFR & AGGAATACATATGGTAGATCAACTCGAAAG CGAAATC \\
Primers used for mutagenesis & AAAGGATCCTCAAGCCCCCTTAGTGAGCAA GCCTCG \\
oAL3 3 & \\
oAL4 & \\
oAL5 & GCGCCCGGGACATCGCCAGGGACTTTCCC \\
oAL6 & TAAGGATCCCCTTTTTTCCAAGGGTTTACGC \\
oAL100 & GTCGGATCCGGCATCACCATCATGCGGTTC \\
oAL101 & CATCTAGACCGCGGTGTTGGAGAGATAACG \\
Other & ACGACATCATCGATGCGACCGGCGTTCTG \\
pet16bup & CAGAACGCCGGTCGCATCGATGATGTC \\
& \\
\hline
\end{tabular}

AcpS, were constructed in an analogous way to that described for pAL22 and pAL24. The resulting plasmids were pAL26 and pTB5001. All coexpression plasmids had the genes for the ACP and for AcpS in the same orientation.

In order to clone smb20650 into pBBR1MCS-5, the primers smb20650R and pet16bup (Table 2) were used to amplify the gene from pAL52. The PCR product was digested with $X b a \mathrm{I}$ and $B g l I I$ and cloned into pBBR1MCS-5 that had been digested with $X b a \mathrm{I}$ and BamHI, resulting in plasmid pAL55.

Site-directed mutagenesis of SMb20651. The single-site mutant of SMb20651 S40A was generated by the PCR overlap extension method (Ho et al., 1989). The oligonucleotide primers oAL3 and oAL101 (Table 2) were used to amplify $1112 \mathrm{bp}$ of genomic DNA spanning from upstream of $s m b 20651$ to 129 bp downstream of the $s m b 20651$ start codon. Similarly, the oligonucleotide primers oAL100 and oAL6 (Table 2) were used to amplify $1003 \mathrm{bp}$ of genomic DNA spanning from $101 \mathrm{bp}$ downstream of the smb20651 start codon to downstream of smb20651. The primers oAL101 and oAL100 overlap in their DNA binding region and introduce a point mutation ( $\mathrm{G}$ to $\mathrm{T}$ ) in nucleotide 118 , causing a serine to alanine substitution in residue 40 of the SMb20651 protein. The two PCR products were used as a template for a new PCR and after heating them for $1 \mathrm{~min}$ at $92{ }^{\circ} \mathrm{C}$, they were annealed by incubation for $4 \mathrm{~min}$ at $60{ }^{\circ} \mathrm{C}$ followed by $6 \mathrm{~min}$ at $72{ }^{\circ} \mathrm{C}$. Then, the oligonucleotide primers smb20651F and smb20651R (Table 2) were added and the PCR was continued following standard procedures. After digestion with $\mathrm{NdeI}$ and BamHI enzymes, the 267 bp product was cloned into pET9a expression vector that had been digested with the same enzymes. The resulting plasmid was named pAL30.

Construction of smb20651 mutants. The oligonucleotide primers oAL3 and oAL4 (Table 2) were used in a PCR to amplify 992 bp of genomic DNA upstream of the smb20651 gene from S. meliloti, introducing SmaI and BamHI sites (underlined in the table) into the
PCR product. Similarly, the primers oAL5 and oAL6 (Table 2) were used to amplify 868 bp of genomic DNA downstream of smb20651, introducing BamHI and $\mathrm{XbaI}$ sites (underlined in the table) into this PCR product. After digestion with the respective enzymes, the $992 \mathrm{bp}$ fragment was cloned into pBluescript SK plasmid that had been digested with SmaI and BamHI, yielding plasmid pAL03. Then, the $868 \mathrm{bp}$ fragment was cloned as a $\mathrm{BamHI} / \mathrm{XbaI}$ fragment into pAL03 plasmid that had been digested with BamHI and XbaI, yielding plasmid pAL04. A spectinomycin-resistance cassette obtained as a BamHI fragment from plasmid pHY109 (Østeras et al., 1998) was inserted into pAL04 that had been digested with BamHI to yield pAL05. Plasmid pAL05 was digested with SmaI and XbaI to reclone the regions usually flanking smb20651 and the spectinomycinresistance gene located between those regions as a SmaI/XbaI fragment into the suicide vector pK18mobsacB (Schäfer et al., 1994) that had been digested with $S m a I$ and $X b a I$ to yield pAL06. Plasmid pAL06 was introduced into wild-type S. meliloti 1021, and double recombinants in which the spectinomycin-resistance cassette replaced smb20651 were obtained following a procedure described previously (Sohlenkamp et al., 2004). The mutation was transduced back into $S$. meliloti 1021 using $\phi \mathrm{M} 12$ as described by Finan et al. (1984), and the mutant strain was named ALR30B1.

Analysis of proteins. Proteins were analysed by $20 \%$ native PAGE (Jackowski \& Rock, 1983) or by conformation-sensitive gels (PostBeittenmiller et al., 1991) containing $16.8 \%$ polyacrylamide in the separation gel and 3.2 M urea in both the separation and stacking gel. Samples were mixed with application buffer to give final concentrations of $7.9 \%(\mathrm{v} / \mathrm{v})$ glycerol before they were loaded onto polyacrylamide gels. Gels were stained with Coomassie blue. Protein concentrations were determined by the method of Dulley \& Grieve (1975) using BSA (Sigma) as standard. For hydroxylamine treatment, proteins were incubated with $100 \mathrm{mM}$ hydroxylamine hydrochloride, $\mathrm{pH} 6.5$, in the presence of $50 \mathrm{mM}$ DTT at $25^{\circ} \mathrm{C}$ for $2 \mathrm{~h}$. 
In vivo labelling of SMb20651 and RkpF with $\left[{ }^{3} \mathrm{H}\right] \boldsymbol{\beta}$-alanine. In vivo labelling of ACPs with $\left[{ }^{3} \mathrm{H}\right] \beta$-alanine, the biosynthetic precursor of $4^{\prime}$-phosphopantetheine, was carried out essentially as described previously using the $\beta$-alanine auxotroph OG7001 (Epple et al., 1998; López-Lara \& Geiger, 2000).

Overexpression of proteins in $\boldsymbol{E}$. coli. The strains were grown at $30{ }^{\circ} \mathrm{C}$ in $500 \mathrm{ml} \mathrm{LB}$ medium supplemented with the appropriate antibiotics. At $\mathrm{OD}_{620} 0.3$, IPTG was added to a final concentration of $100 \mu \mathrm{M}$. Cells were harvested by centrifugation and cell pellets were resuspended either in ice-cold buffer A $(50 \mathrm{mM}$ Tris/ $\mathrm{HCl}, \mathrm{pH} 6.8)$, or in ice-cold start buffer $(20 \mathrm{mM}$ sodium phosphate, $0.5 \mathrm{M} \mathrm{NaCl}$, $10 \mathrm{mM}$ imidazole, $\mathrm{pH}$ 7.4) when used for subsequent chromatography on $\mathrm{Ni}^{2+}$ agarose. The cells were broken by three passages through a French press at $138 \mathrm{MPa}$. Broken cell suspensions were centrifuged for $10 \mathrm{~min}$ at $15550 \mathrm{~g}$ and the supernatants were recovered. His-AcpS was overproduced as inclusion bodies, in which case the pellet was recovered after centrifugation.

Purification of SMb20651 and NodF. Cold 2-propanol was added dropwise to the cell-free protein extracts until a final concentration of $50 \%(\mathrm{v} / \mathrm{v})$ was reached. After incubation for $90 \mathrm{~min}$, the precipitate was removed by centrifugation at $15550 \mathrm{~g}$ for $20 \mathrm{~min}$ and the final 2propanol concentration was adjusted to $10 \%$ with buffer A. The supernatant was then applied to a $5 \mathrm{ml}$ DEAE-52 cellulose (Whatman) column, which had been equilibrated with buffer $\mathrm{B}$ ( $50 \mathrm{mM}$ Bistris $/ \mathrm{HCl}, \mathrm{pH} 6.8$ ). The column was washed with $25 \mathrm{ml}$ buffer B and elution was performed with a step-wise gradient of 0.2 , $0.5,1$ and $2 \mathrm{M} \mathrm{NaCl}$ in buffer B. Fractions $(1 \mathrm{ml})$ were collected and aliquots were analysed by native or conformation-sensitive PAGE as required. The SMb20651, NodF and acylated SMb20651 proteins eluted with the addition of $0.5 \mathrm{M} \mathrm{NaCl}$. Fractions containing the respective ACP were individually applied to a PrepCell (model 491, Bio-Rad) containing a cylindrical $20 \%$ native polyacrylamide gel in the case of SMb20651 and NodF, and a $16.8 \%$ polyacrylamide gel containing $3.2 \mathrm{M}$ urea in the case of acylated SMb20651. Fractions ( $3 \mathrm{ml}$ each) were collected and SMb20651- or NodF-containing fractions were concentrated by ultrafiltration at $4{ }^{\circ} \mathrm{C}$. The buffer was changed to $10 \mathrm{mM}$ Tris/ $\mathrm{HCl}, \mathrm{pH} 8.0$, for enzymic preparation of holo-SMb20651 and holo-NodF. Samples containing acylated SM20651 were dried and analysed for fatty acid content at the Bacterial Identification \& Fatty Acid Analysis Laboratory, Plant Pathology Department, University of Florida, Gainsville.

For large-scale SMb20651 enrichment from S. meliloti, 101 cultures were grown on TY at $30{ }^{\circ} \mathrm{C}$ to $\mathrm{OD}_{620}$ 0.7. Purification steps were as described above with the following differences: cold 2-propanol was added to $30 \%(\mathrm{v} / \mathrm{v})$ final concentration and elution was performed with a linear gradient $(0-2 \mathrm{M} \mathrm{NaCl})$ from a DEAE-Sepharose column (Pharmacia Biotech). The fractions containing SMb20651 were pooled and passed through a YM-50 filter device with a molecular weight cutoff of $50000 \mathrm{Da}$. Then, the filtrate was desalted and concentrated with a YM-3 filter device with a molecular weight cutoff of $3000 \mathrm{Da}$. A similar mock purification was carried out using smb20651-deficient-strain ALR30B1.

Purification of His-tagged proteins. The cell-free crude extract of the E. coli strain overproducing His-SMb20651 was first fractionated with $60 \%$ 2-propanol, and the 2-propanol was then eliminated by rotatory evaporation. The inclusion bodies consisting of His-AcpS protein were washed twice in start buffer, then dissolved in start buffer containing $6 \mathrm{M}$ guanidine hydrochloride and centrifuged at $6300 \mathrm{~g}$. The samples containing His-SMb20651 or AcpS prepared as described above or the cell-free extract of the strain overproducing His-FabD were applied to a $1 \mathrm{ml} \mathrm{Ni}^{2+}$ agarose column (Hi Trap, Pharmacia Biotech). $\mathrm{Ni}^{2+}$ chromatography was performed according to the manufacturer's instructions. In the case of His-AcpS all buffers contained $6 \mathrm{M}$ guanidine hydrochloride. The three proteins were eluted with $500 \mathrm{mM}$ imidazole in start buffer. After concentration with Amicon devices (Millipore), aliquots of purified His-AcpS and His-FabD were kept in sodium phosphate buffer containing $14.3 \%$ $(\mathrm{v} / \mathrm{v})$ glycerol at $-80{ }^{\circ} \mathrm{C}$.

Generation of polyclonal antiserum against the ACP SMb20651 and Western blot analysis. Antiserum against purified HisSMb20651 was raised in a white New Zealand rabbit that had received four injections, each of a 1:1 mixture of Freud's complete or incomplete adjuvant (Roche) together with $150 \mu \mathrm{g}$ His-SMb20651 antigen protein in $137 \mathrm{mM} \mathrm{NaCl}, 2.7 \mathrm{mM} \mathrm{KCl}, 4.3 \mathrm{mM} \mathrm{Na}_{2} \mathrm{HPO}_{4}$ and $1.4 \mathrm{mM} \mathrm{KH}_{2} \mathrm{PO}_{4}$.

For immunodetection of SMb20651, protein samples were separated by PAGE and electrophoretically transferred to a nitrocellulose membrane (Hybond-C Extra, Amersham Biosciences). Membranes were blocked with $5 \%$ non-fat milk in PBS $(150 \mathrm{mM} \mathrm{NaCl}$, sodium phosphate, $\mathrm{pH}$ 7.2) and then incubated with anti-His-SMb20651 serum diluted either $1: 1000$ or $1: 100$ for E. coli- or S. melilotiderived protein samples, respectively. Anti-rabbit immunoglobulin antibodies (alkaline phosphatase conjugate) were used as secondary antibodies. Reaction results were visualized with Sigma Fast 5-bromo4-chloro-3-indolyl phosphate/nitro blue tetrazolium tablets according to the manufacturer's recommendations.

In vitro modification of apo-ACPs to holo-ACPs. For preparation of holo-SMb20651 and holo-NodF, the purified proteins were incubated with CoA in the presence of $S$. meliloti His-AcpS. Reactions were performed in $10 \mathrm{mM}$ Tris $/ \mathrm{HCl}, \mathrm{pH} 8.0$, containing $10 \mathrm{mM}$ DTT, $5 \mathrm{mM} \mathrm{MgCl}_{2}, 300 \mu \mathrm{M}$ CoA, $40.6 \mu \mathrm{M}$ ACP and $2.3 \mu \mathrm{M}$ His-AcpS in a final volume of $50 \mu \mathrm{l}$. Reactions were incubated at $30{ }^{\circ} \mathrm{C}$ for $4 \mathrm{~h}$. MALDI-TOF MS was performed using a Bruker Daltonics Autoflex mass spectrometer operated in the linear mode. Spectra were externally calibrated using a protein calibration standard (Bruker Daltonics 206295). Protein mixtures were desalted using a C18 ZipTip (Millipore) and analysed using a saturated solution of sinapinic acid in $50 \%(\mathrm{v} / \mathrm{v})$ acetonitrile and $0.1 \%(\mathrm{v} / \mathrm{v})$ trifluoroacetic acid.

In vitro malonylation of holo-SMb20651 and holo-NodF. Reactions were performed in $50 \mathrm{mM}$ sodium phosphate buffer, $\mathrm{pH} 7.2$, containing $2 \mathrm{mM}$ DTT, $2 \mathrm{mM}$ EDTA, $0.1 \mathrm{mM} \mathrm{FeSO}_{4}$, $25 \mathrm{mM} \mathrm{MgSO}_{4}, 17 \mu \mathrm{M}$ ACP, $6.2 \mu \mathrm{M}$ His-FabD and $1 \mathrm{mM}$ malonyl-CoA in a final volume of $30 \mu \mathrm{l}$. Reactions were incubated at $30{ }^{\circ} \mathrm{C}$ for $1 \mathrm{~h}$. In assays involving radioactive malonyl-CoA, $6105 \mathrm{~Bq}$ $\left[{ }^{14} \mathrm{C}\right]$ malonyl-CoA (specific activity $4.07 \times 10^{8} \mathrm{~Bq} \mathrm{mmol}^{-1}$; American Radiolabelled Chemicals) were used per reaction in a final volume of $15 \mu$ l. Malonyl-ACP samples were separated by conformationsensitive PAGE. Radiolabelled samples were also transferred to a nitrocellulose membrane, and the incorporation of the malonyl group was demonstrated by autoradiography.

Filter-disk assays. Zones-of-inhibition assays were performed as described elsewhere (Ferguson et al., 2002). A $5 \mu$ laliquot of the stock agents $10 \%$ SDS, $70 \mathrm{mg}$ gentamicin $\mathrm{ml}^{-1}, 2 \%$ sodium deoxycholate (DOC), $\quad 10 \%$ cetyltrimethylammoniumbromide (CTAB), $10 \%$ Triton X-100 or $10 \%$ Brij 58 was applied to the disk. For the polymyxin B assays, commercial test disks (300 units per disc, Oxoid) were used. At least three repetitions were made for each agent tested. Zones of inhibition were measured after 2 days of growth at $30^{\circ} \mathrm{C}$.

Plant assays. Alfalfa (Medicago sativa L.) plants were grown in a nitrogen-free medium as described elsewhere (Olivares et al., 1980). Nitrogen fixation was determined by examining acetylene reduction using GC (Burris, 1972). 


\section{RESULTS}

\section{smb20651 is part of a four-gene operon}

smb20651 is located on plasmid pSymB as part of a possible operon of four genes (Fig. 1). We designed primers to amplify the intergenic regions of the adjacent genes, using RT-PCR. Three intergenic regions between adjacent genes (asnB, smb20651, smb20650 and nadE1) were amplified (Supplementary Fig. S1) from the RNA samples of $S$. meliloti 1021 cells grown on TY. These results indicate that the four genes (asnB, smb20651, smb20650 and nadE1) form an operon.

Interestingly, while the same operon is conserved in the genomes of the rhizobial species Sinorhizobium medicae WSM419, Mesorhizobium sp. BNC1 and Mesorhizobium huakuii MAFF 303099 (Fig. 1), it is absent in the genomes of rhizobia from the genera Rhizobium, Bradyrhizobium and Azorhizobium. The four genes forming the operon are also present in the $\alpha$-proteobacterium Rhodopseudomonas palustris BisA53. Remarkably, the operon is also found in phylogenetically unrelated bacteria such as Planctomyces, Roseiflexus and Geobacter, and even in the archaeon Methanosarcina (Fig. 1). Parts of the operon are conserved in other species, and the most frequent association is between the genes encoding the putative ACP and a potential long-chain fatty acyl-CoA ligase (Fig. 1), suggesting that SMb20650 homologues could be involved in loading the respective putative SMb20651 ACPs with acyl chains.

\section{Efficient phosphopantetheinylation of SMb20651 occurs only in the presence of extra AcpS}

ACPs are functional when substituted with the $4^{\prime}$ phosphopantetheine prosthetic group. Cell-free extracts of E. coli OG7001(pLysS) carrying either empty pET9a, or plasmids that allow overproduction of SMb20651 or RkpF that had been grown in the presence of $\left[{ }^{3} \mathrm{H}\right] \beta$-alanine, a biosynthetic precursor of $4^{\prime}$-phosphopantetheine, were analysed by PAGE and subsequent autoradiography. Although the SMb20651 protein was highly overproduced (Fig. 2a, lane 2), incorporation of $\left[{ }^{3} \mathrm{H}\right] \beta$-alanine was not detected (Fig. 2b, lane 2), indicating poor or lack of holo$\mathrm{SMb} 20651$ production. In contrast, efficient production of holo-RkpF was detected (Fig. 2b, lane 6). ACPs are phosphopantetheinylated by enzymes of the phosphopantetheinyl transferase (PPT) family. To test whether SMb20651 requires S. meliloti PPT AcpS (SMc02654) for modification, we constructed plasmids that allow the coexpression in cis of SMb20651 together with either $E$. coli AcpS or S. meliloti AcpS. As controls, we made the same constructions with RkpF, which is an ACP from $S$. meliloti Rm41 (Epple et al., 1998). Although under coexpression conditions we detected much less SMb20651 or RkpF protein (Fig. 2a, lanes 1, 3, 5 and 7), both proteins were efficiently labelled with $\left[{ }^{3} \mathrm{H}\right] \beta$-alanine (Fig. 2b, lanes 1, 3, 5 and 7). Surprisingly, the over-
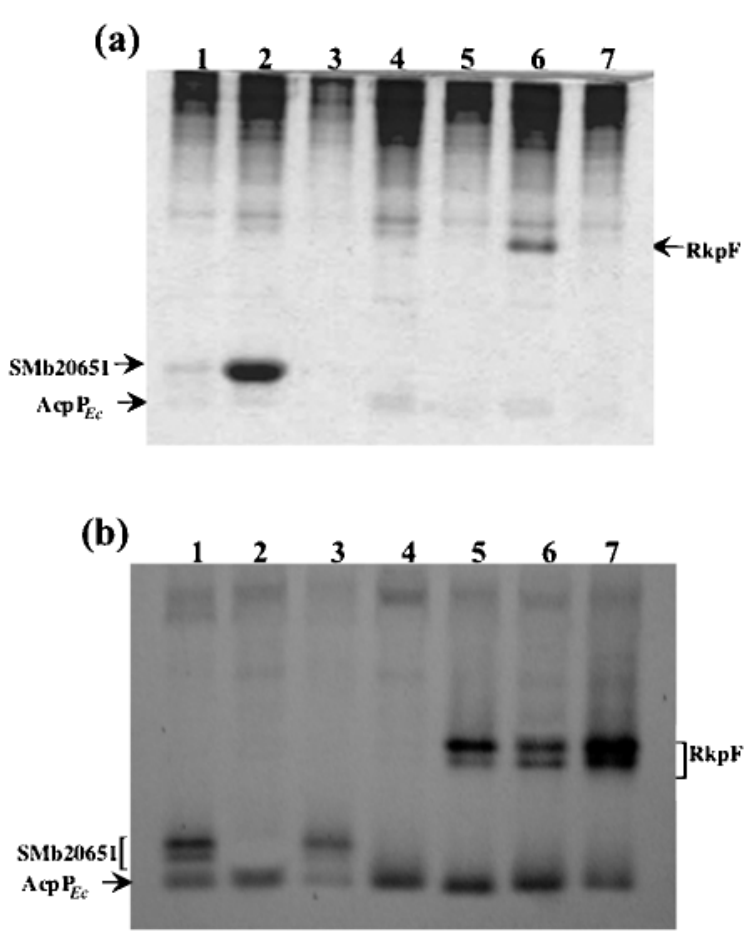

Fig. 2. Overproduction of S. meliloti ACPs in E. coli OG7001 (pLysS). Analysis of cell-free extracts from E. coli strains overproducing SMb20651 and RkpF. (a) Proteins separated by $20 \%$ native PAGE and stained with Coomassie blue. (b) Autoradiogram after labelling with $\left[{ }^{3} \mathrm{H}\right] \beta$-alanine. $(\mathrm{a}, \mathrm{b})$ Cell-free extracts of $E$. coli overproducing: SMb20651 and $\operatorname{AcpS}_{E c}$ (lane 1), SMb20651 (lane 2), SMb20651 and $\mathrm{AcpS}_{S m}$ (lane 3), nothing (E. coli strain carrying pET9a) (lane 4), RkpF and $\operatorname{AcpS}_{E_{c}}$ (lane 5), RkpF (lane 6), and $\mathrm{RkpF}$ and $\mathrm{AcpS}_{S m}$ (lane 7). The bands corresponding to $\mathrm{SMb} 20651, \mathrm{RkpF}$ and the E. coli AcpP are indicated.

expression of either AcpS of S. meliloti or AcpS of E. coli can modify SMb20651, indicating that the level of AcpS in wild-type E. coli is not enough to modify SMb20651, or that E. coli AcpS does not work efficiently with SMb20651. The latter could be due to the presence of the sequence motif DST (where $S$ is the 4 '-phosphopanteteine binding site) instead of the DSL sequence present in E. coli and other sinorhizobial ACPs (Supplementary Fig. S2).

\section{Serine 40 is necessary for $4^{\prime}$-phosphopantetheine binding to SMb20651}

SMb20651 carrying the S40A change in the conserved serine of the predicted 4 '-phosphopantetheine binding site was coexpressed in trans together with S. meliloti AcpS, and in vivo labelling with $\left[{ }^{3} \mathrm{H}\right] \beta$-alanine was performed. The amount of SMb20651 (S40A) present in the crude extract was similar to that found when SMb20651 is overexpressed alone (Fig. 3a, b, lane 4) but it could no longer be labelled with $\left[{ }^{3} \mathrm{H}\right] \beta$-alanine (Fig. $3 \mathrm{c}$, lane 4 ), indicating that serine 40 is necessary for $4^{\prime}$-phosphopantetheine binding. SMb20651 (S40A) migrates similarly in native PAGE to 
(a)

(b)

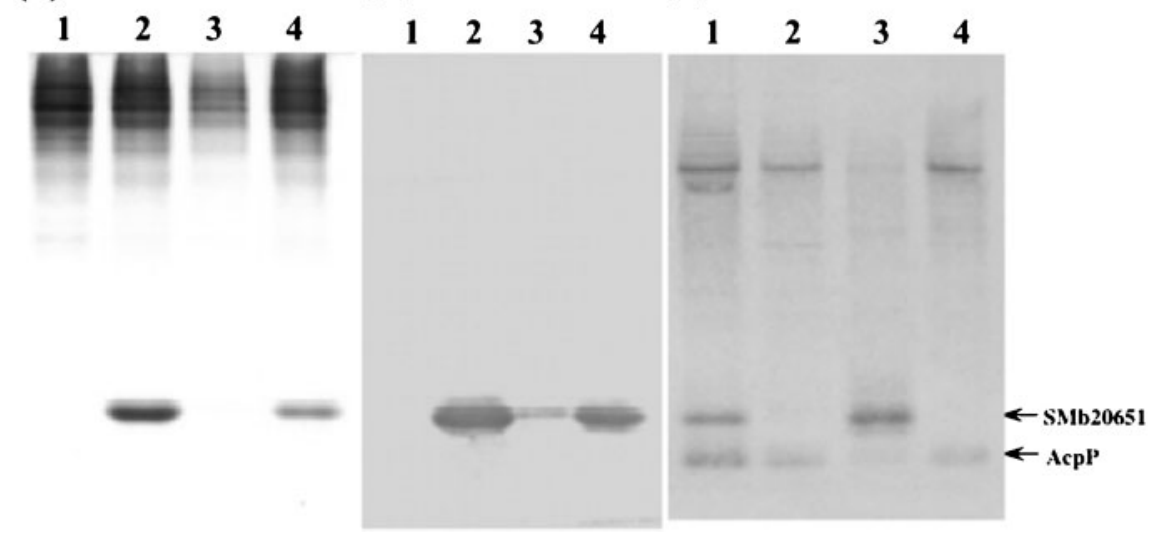

Fig. 3. Serine 40 is required for $4^{\prime}$-phosphopantetheinylation of SMb20651. (a) Analysis of cell-free extracts from $E$. coli strains overproducing SMb20651. Proteins were separated by $20 \%$ native PAGE and stained with Coomassie blue. (b) Western blot detection of SMb20651 using anti-His-SMb20651 antibodies. (c) Autoradiogram of cell-free extracts of E. coli strains overproducing SMb20651 after labelling with $\left[{ }^{3} \mathrm{H}\right] \beta$-alanine. In (a), (b) and (c), cell-free extracts of E. coli are shown overproducing SMb20651 and $A_{c p S}$ in cis (lane 1), SMb20651 (lane 2), SMb20651 and AcpS $S_{S m}$ in trans (lane 3), and $\mathrm{SMb20651(S40A)}$ and $\mathrm{AcpS}_{S m}$ in trans (lane 4). Overexpression in trans was achieved by expressing in E. coli derivatives of pET9a and pBBR1MCS-5 carrying acp $S_{S m}$ (pAL41). The bands corresponding to SMb20651 and E. coli AcpP are indicated.

wild-type SMb20651, and therefore the exchange S to A probably does not affect the overall folding and conformation of this protein (Fig. 3, lane 4).

\section{In vitro 4'-phosphopantetheine transfer to SMb20651}

Analysis of purified SMb20651 by MALDI-TOF MS showed an $m / z$ of 9307.3 and one of 9645.5 for
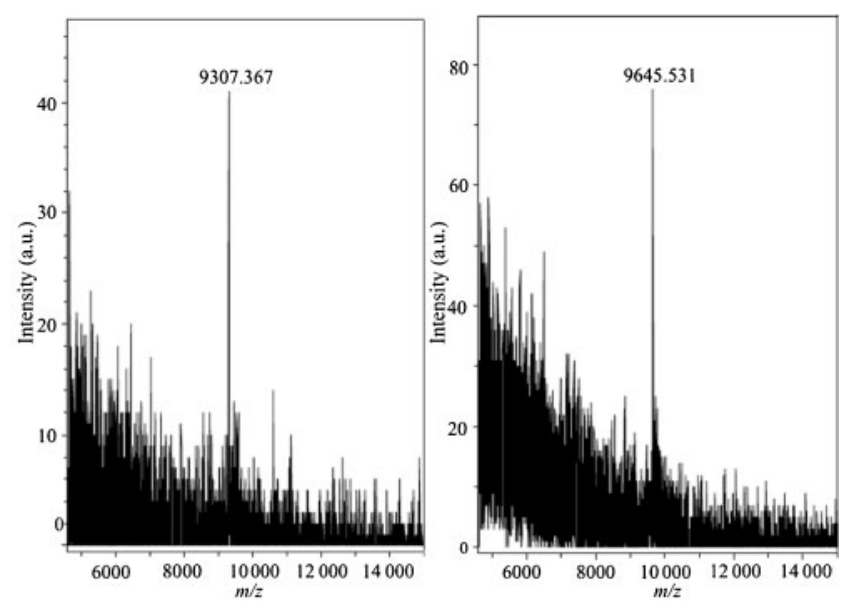

Fig. 4. In vitro 4'-phosphopantetheinylation of SMb20651 observed by MALDI-TOF MS. Spectra of SMb20651 incubated with $\mathrm{CoA}$ in the absence (a) or presence (b) of purified $S$. meliloti His-AcpS. Intensity is given in arbitrary units (a.u.).
SMb20651 that had been incubated with His-AcpS (Fig. $4)$. These values are in close agreement with the predicted masses for apo-SMb20651 (9305.51) and holo-SMb20651 (9644.51) lacking the initial methionine. The difference of 338.2 atomic mass units (a.m.u.) is consistent with the expected 339 a.m.u. for the $4^{\prime}$-phosphopantetheine addition, indicating that His-AcpS-treated SMb20651 is carrying the prosthetic group typical of ACPs. Therefore, AcpS attaches $4^{\prime}$-phosphopantetheine to SMb20651.

\section{SMb20651 is malonylated by FabD}

Malonyl CoA:ACP transacylase (FabD) is the enzyme responsible for the transfer of the malonyl group from malonyl-CoA to holo-ACP. The resulting product is an intermediate in the initiation and elongation steps during fatty acid and polyketide synthesis. We examined the in vitro transfer of the malonyl moiety from malonyl-CoA to SMb20651 by $S$. meliloti His-FabD. Conformation-sensitive electrophoresis of the reaction products shows a slowermigrating form of SMb20651 that appeared only when the protein was incubated with malonyl-CoA (Fig. 5a, lanes 1 and 2). It has been shown that NodF from Rhizobium leguminosarum bv. viciae can be malonylated in vitro using the enzyme FabD from E. coli (Ritsema et al., 1998), and therefore we used $S$. meliloti NodF as a positive control in this study. Although no conformational change was observed when $\operatorname{NodF}_{S m}$ was incubated with malonyl-CoA and $\mathrm{FabD}_{S m}$ (Fig. 5b, lanes 1 and 2), the radioactive assays confirmed the incorporation of the malonyl group into both ACPs (Fig. 5, lanes 4). 


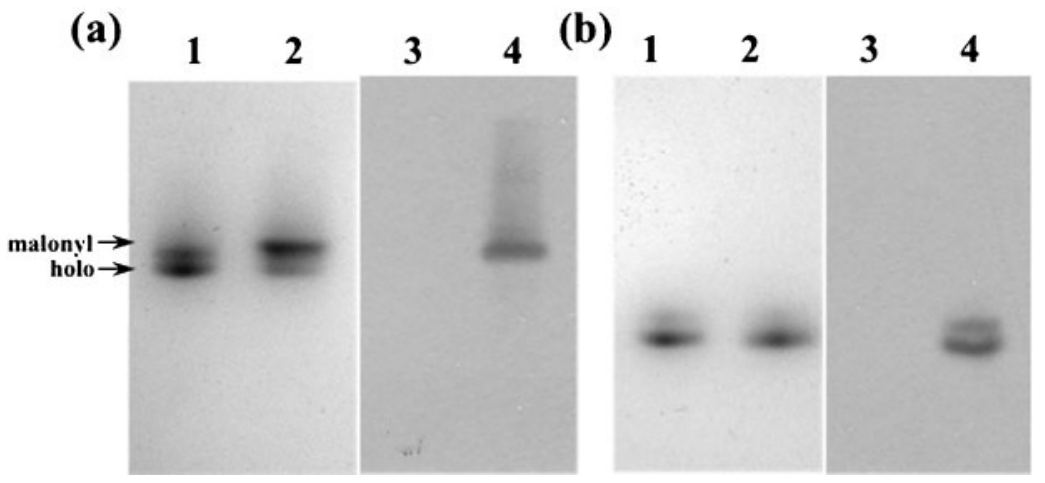

Fig. 5. Malonylation of SMb20651 (a) or NodF (b) by FabD. Proteins were separated by conformation-sensitive PAGE. $(a, b)$ Lanes 1 and 2, Coomassie blue-stained proteins; lanes 3 and 4 , autoradiogram. (a, b) The proteins are: holo-ACP assayed without (lane 1) or with malonyl-CoA (lane 2), holo-ACP assayed without (lane 3 ) or with $\left[{ }^{14} \mathrm{C}\right]$ malonylCoA (lane 4). The positions of malonylSMb20651 (malonyl) and holo-SMb20651 (holo) are indicated.

\section{SMb20651 is modified in $E$. coli when it is coexpressed with $S$. meliloti enzymes}

SMb20650 is a predicted long-chain fatty acyl-CoA ligase and its gene is cotranscribed with the gene for SMb20651. Although apo- and holo-SMb20651 show the same mobility in $20 \%$ native PAGE, they run significantly differently in conformation-sensitive gels (Fig. 6, lanes 1 and 2), and possible acylated forms are expected to have different mobilities in this type of gel (Post-Beittenmiller et al., 1991). To test whether SMb20650 could be involved in the acylation of SMb20651, we coexpressed in E. coli the protein SMb20651 together with AcpS Sm $_{\text {and SMb20650 }}$ (by means of the plasmids pAL22 and pAL55). As controls, we expressed SMb20651 alone or together with $\mathrm{AcpS}_{S m}$ using plasmid pAL22. After DEAE-52 cellulose purification, the resulting proteins were analysed by conformation-sensitive PAGE. The E. coli strain carrying plasmid pAL20651 produces apo-SMb20651 (Fig. 6, lane 4). Surprisingly, besides the expected holo form (mainly migrating as a dimer), possible modified SMb20651 species were also detected when the protein was coexpressed with AcpS alone (Fig. 6, lane 5). When SMb20651 was coexpressed with AcpS and SMb20650, we detected two additional forms that are possibly acylSMb20651 (indicated by asterisks in Fig. 6, lane 6). The presence of the modification makes the proteins more compact so that they run significantly faster in these gels. After hydroxylamine treatment, which is known to cleave thioester linkages, all the possible modifications on SMb20651 disappeared, while holo-SMb20651 was increased (Fig. 6a, lanes 8 and 9), indicating that the hydroxylamine-sensitive forms of SMb20651 carry thioester-linked acylations. The identity of the proteins detected in E. coli as SMb20651 species was confirmed by Western blot analysis (Fig. 6b). These results suggest that E. coli enzymes can modify holo-SMb20651. GC analysis of CellPrep-purified SMb20651 obtained from $E$. coli BL21(DE3) (pLysS) (pAL22) (pAL55) revealed the presence of $\mathrm{C} 12: 0$ and $\mathrm{C} 18: 0$ fatty acids that were not detected in a similarly purified fraction obtained from the strain E. coli BL21(DE3) (pLysS) (pET9a) (data not shown).

\section{Different forms of SMb20651 are detected in S. meliloti 1021}

SMb20651 is expressed in S. meliloti 1021 growing in complex TY and in minimal M9 medium in the exponential and stationary phases of growth (data not shown). SMb20651 was partially purified by DEAESepharose chromatography from cell-free extracts obtained from S. meliloti 1021 cultures, and a similar mock purification was carried out from cell-free extracts of $S$. meliloti ALR30B1 as a control. When the S. meliloti SMb20651-enriched fractions were analysed by conformation-sensitive PAGE and tested with anti-His-SMb20651, five different SMb20651 forms were detected (Fig. 7b, lane 4). Presumably these forms could be holo- and modified species of holo-SMb20651. Two of these SMb20651 forms migrate slower than the possible holo-SMb20651; the other two migrate faster than the possible holo-SMb20651. No signal was detected in the mock purification of the control ALR30B1 (Fig. 7b, lanes 6 and 7). After hydroxylamine treatment, the possible modified proteins disappeared, while holo-SMb20651 was increased (Fig. 7b, lane 5). These results strongly indicate that SMb20651 is acylated in $S$. meliloti. Interestingly, the protein band indicated as $\mathrm{X}$ in lanes 4 and 5 (Fig. 7a) was obtained only in the enrichment procedure with S. meliloti 1021. This protein is not recognized by anti-His-SMb20651, is not sensitive to hydroxylamine treatment and is detected only in the presence of SMb20651. After hydroxylamine treatment of enriched samples of either wild-type or SMb20651 mutant, a fast doublet migrating band appeared (Fig. 7a, lanes 5 and 7). This band is probably the result of deacylation of different AcpP forms, since these doublets migrate similarly to purified holo-AcpP from S. meliloti 1021 (Fig. 7a, lane 8). From the results shown in lane 5 (Fig. 7a), it can be concluded that SMb20651 is far less abundant than AcpP, which is known to be one of the most abundant soluble proteins in the bacterial cell.

\section{Phenotypic characterization of SMb20651 mutants}

The mutant ALR30B1 presented no growth differences with respect to the wild-type 1021 when grown on TY 
(a)

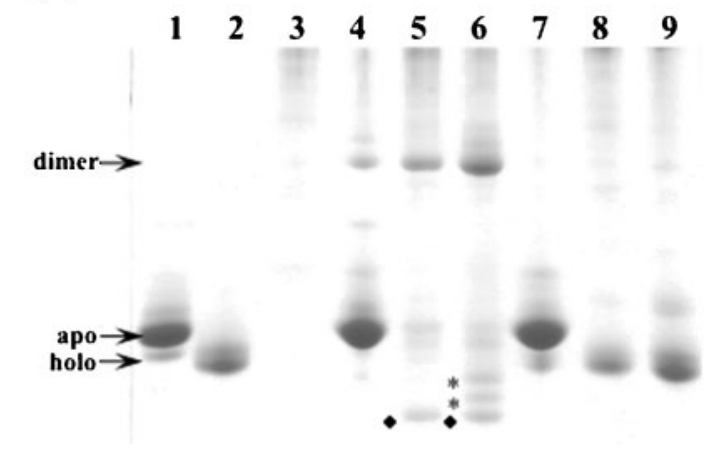

(b)

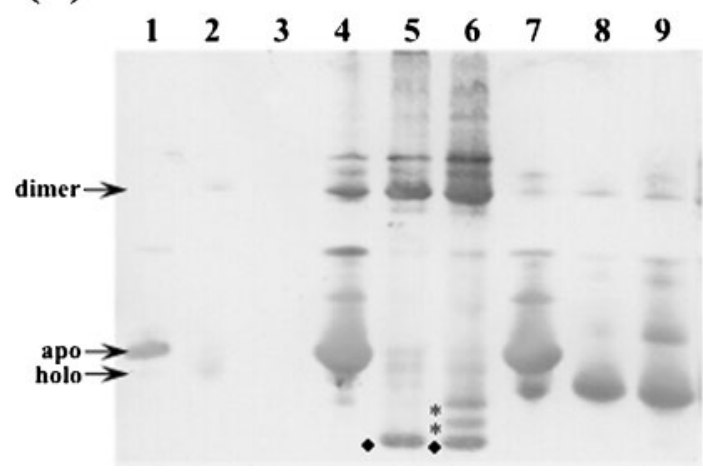

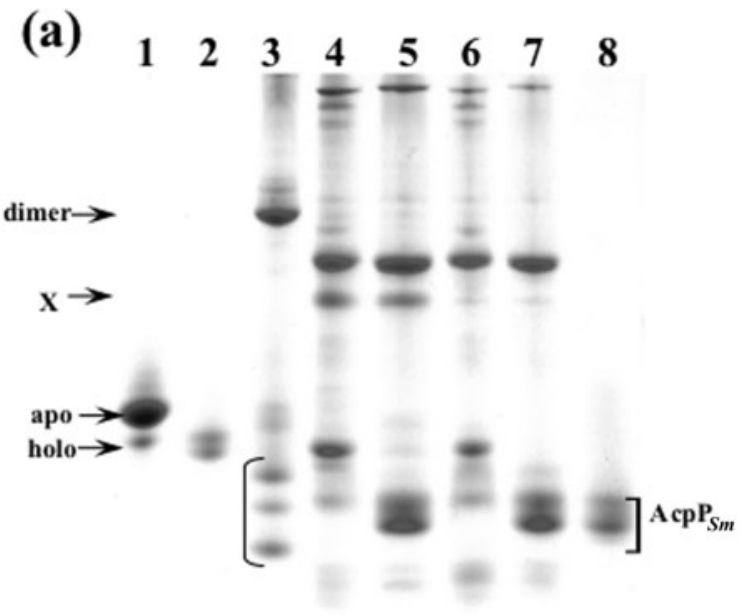

(b)

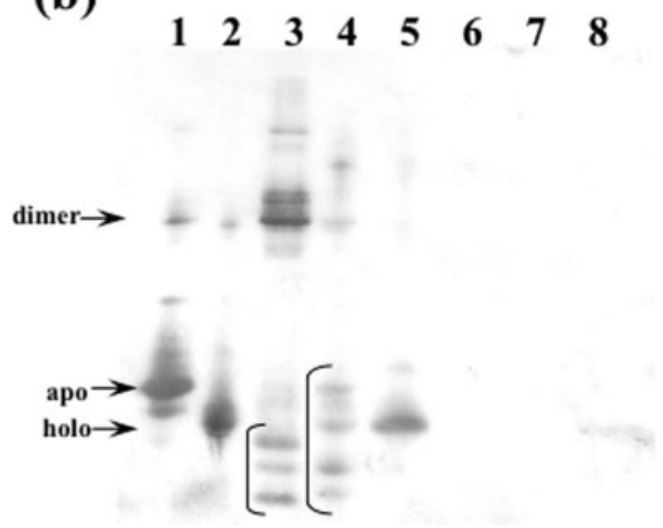

Fig. 6. Analysis of DE52-enriched SMb20651 proteins from different E. coli BL21(DE3) (pLysS) host strains. Proteins were partially purified by anion exchange on a DE52-cellulose column and separated by conformation-sensitive PAGE. (a) Coomassie blue staining of proteins. (b) Western blot detection using anti HisSMb20651 antibodies. The proteins analysed are: purified apoSMb20651 (lane 1), purified holo-SMb20651 (lane 2); enrichment from E. coli BL21(DE3) (pLysS) overexpressing: nothing (empty pET9a plasmid) (lane 3), SMb20651 (lane 4), SMb20651 and AcpS $_{S m}$ (lane 5), SMb20651, AcpS $S_{S m}$ and SMb20650 (lane 6); lanes 7, 8 and 9 contain the same samples as in lanes 4, 5 and 6, respectively, after hydroxylamine treatment. The two asterisks in lane 6 indicate the bands specifically formed after coexpresion of the three proteins in E. coli, while the diamonds in lanes 5 and 6 indicate the band formed after coexpression of SMb20651 and AcpS. The positions of the dimer, apo and holo forms of SMb20651 are indicated.

medium or in any of the minimal media (M9, Robertsen or Sherwood). In addition, no differences were found when using different nitrogen sources for growth or TY medium with different concentrations of $\mathrm{NaCl}$. Using filter-disk assays, no difference was found in sensitivity to the detergents SDS, DOC, CTAB, Triton X-100 and Brij 58, nor to the antibiotics gentamicin and polymyxin.

Alfalfa plants were inoculated with either ALR30B1 or the parental 1021 strain, and no significant differences in the number of nodules or in the course of nodule development
Fig. 7. Analysis of DEAE-enriched proteins from S. meliloti 1021 and ALR30B1. Proteins were separated by conformation-sensitive PAGE. (a) Coomassie blue staining of proteins. (b) Western blot detection using anti His-SMb20651 antibodies. The proteins analysed are: purified apo-SMb20651 (lane 1), purified holoSMb20651 (lane 2), enrichment from E. coli BL21(DE3) pLysS overexpressing SMb20651, $\mathrm{AcpS}_{\mathrm{Sm}}$ and SMb20650 (lane 3), enrichment from S. meliloti 1021 (lane 4), enrichment from $S$. meliloti 1021 followed by hydroxylamine treatment (lane 5), enrichment from $S$. meliloti ALR30B1 (lane 6), enrichment from S. meliloti ALR30B1 followed by hydroxylamine treatment (lane 7), purified holo-AcpP $\mathrm{Sm}_{\mathrm{S}}$ (lane 8). $\mathrm{X}$ indicates a protein band in lanes 4 and 5 that is present only in the enrichment from wild-type $S$. meliloti. The running positions of the dimer, apo and holo forms of SMb20651 as well as S. meliloti AcpP $\left(\operatorname{AcpP}_{S m}\right)$ are indicated. The brackets in lanes 3 and 4 enclose different forms of SMb20651 after overexpression of three proteins in E. coli and those observed in wild-type $S$. meliloti, respectively.

were observed. After 40 days, the nitrogen-fixing ability was measured and no significant differences were observed.

\section{DISCUSSION}

S. meliloti has four well-characterized ACPs, one for the synthesis and transfer of essential fatty acids, and three 
specialized ACPs whose functions are related to symbiotic interaction between rhizobia and their host plants (Geiger \& López-Lara, 2002). Here, we present the characterization of SMb20651 as the fifth functional ACP of S. meliloti. Importantly, SMb20651 can be loaded with a malonyl residue, and the malonyl-ACP product is the building block in fatty acid synthesis and in the synthesis of some polyketides. In cultures of S. meliloti, holo and acylated forms of SMb20651 were detected, indicating that SMb20651 is metabolically active, probably in the formation and transfer of a fatty acid for which the function or final target molecule is not yet known. So far, the relevance of this pathway cannot be established, since we could not find any phenotype associated with the deletion of SMb20651, either in free-living bacteria or in symbiosis with alfalfa plants. In earlier expression profile experiments (Becker et al., 2004), it has been found that both smb20651 and smb20650 are considerably repressed during symbiosis. Also, nadE1, smb20650 and smb20651 are repressed under iron-limiting conditions (Chao et al., 2005). In another study, Domínguez-Ferreras et al. (2006) showed repression of smb20651 and smb20650 after osmotic shock with $\mathrm{NaCl}$, although in RT-PCR experiments smb20651 was induced twofold. While all these data confirm that smb20651 and smb20650 are part of an operon and that their products might function together, they offer no clue to the possible function of SMb20651. smb20651 is part of an operon with $a s n B$ (encoding a putative asparagine synthetase) and nadE1 (encoding a putative ammonium-dependent $\mathrm{NAD}^{+}$ synthetase). However, the operon is not essential for asparagine or NAD synthesis, since the mutant in smb20651 can grow in minimal media.

The coexpression of SMb20651 with either E. coli AcpS or S. meliloti AcpS (SMc02654) leads to efficient in vivo labelling with $\left[{ }^{3} \mathrm{H}\right] \beta$-alanine, but formation of holoSMb20651 goes in parallel with reduced growth of the expressing strain and a remarkable reduction of the total amount of ACP present. The source of this seemingly toxic effect is probably the products formed by the coexpression, because expression of either SMb20651 alone or of AcpS together with SMb20651 (S40A), which cannot be phosphopantetheinylated, does not cause growth inhibition. Furthermore, we have observed that after coexpression of SMb20651 and AcpS in E. coli, a significant proportion of SMb20651 is modified not only to the holo form but also to acylated forms (Fig. 6). It is possible that by means of this modification the E. coli strain is being depleted of some essential substrates. The toxic effect is also observed after coexpression of RkpF and AcpS, and it would be interesting to study the molecular mechanism that causes this toxicity.

The overall amino acid sequence identity of SMb20651 to the other ACPs of S. meliloti ranges from 10 to $31 \%$, and a conserved amino acid region can only be observed around the phosphopantetheine binding site (Supplementary Fig. S2). With $31 \%$ identity, AcpP is the most similar of the $S$. meliloti ACPs to SMb20651. However, among the ACP homologoes to SMb20651, regions of conserved amino acids are observed along the whole sequence of the proteins and cluster mainly in three regions (Supplementary Fig. S2). If SMb20651 is most similar to AcpP, it might replace AcpP in some of its functions.

SMb20650 is predicted to be a long-chain fatty acyl-CoA ligase (FadD), which activates acyl groups to acyl-CoAs. However, our results indicate that SMb20650 plays a role in the transfer of a specific product, presumably a longchain fatty acid, to the ACP SMb20651. The soluble acylACP synthetase (AasS) of Vibrio harveyi B392 links fatty acids to ACP and is a member of the medium-chain acylCoA synthetase family (Jiang et al., 2006). AasS of $V$. harveyi shows only $25 \%$ identity to SMb20650 and therefore they are only distantly related.

\section{ACKNOWLEDGEMENTS}

We are grateful to R. J. Cox, University of Bristol, for providing pRJC005. We thank Sandra L. Beltrán-Escobar for constructing plasmids pSBA01 and pSBD02. We are indebted to Miguel Angel Cevallos and Nicolás Gómez-Hernández for their invaluable help with the RT-PCR experiments. This work was supported by a grant from CONACyT/Mexico (49738-Q) with partial support from the Howard Hughes Medical Institute (HHMI 55003675). A. L. R.-V. was supported during the $\mathrm{PhD}$ program (Programa de Doctorado en Ciencias Biomédicas, Universidad Nacional Autonoma de México) by scholarships from Consejo Nacional de Ciencia y Tecnología (Mexico) and Dirección General de Estudios de Postgrado (UNAM).

\section{REFERENCES}

Altschul, S. F., Madden, T. L., Schäfer, A. A., Zhang, J., Zhang, Z., Miller, W. \& Lipman, D. J. (1997). Gapped BLAST and PSI-BLAST: a new generation of protein database search programs. Nucleic Acids Res 25, 3389-3402.

Becker, A., Bergès, H., Krol, E., Bruand, C., Rüberg, S., Capela, D., Lauber, E., Meilhoc, E., Ampe, F. \& other authors (2004). Global changes in gene expression in Sinorhizobium meliloti 1021 under microoxic and symbiotic conditions. Mol Plant Microbe Interact 17, 292-303.

Beringer, J. E. (1974). R factor transfer in Rhizobium leguminosarum. J Gen Microbiol 84, 188-198.

Brozek, K. A., Carlson, R. W. \& Raetz, C. R. (1996). A special acyl carrier protein for transferring long hydroxylated fatty acids to lipid A in Rhizobium. J Biol Chem 271, 32126-32136.

Burris, R. H. (1972). Nitrogen fixation assay-methods and techniques. Methods Enzymol 24, 415-431.

Byers, D. M. \& Gong, H. (2007). Acyl carrier protein: structurefunction relationships in a conserved multifunctional protein family. Biochem Cell Biol 85, 649-662.

Chao, T. C., Buhrmester, J., Hansmeier, N., Pühler, A. \& Weidner, S. (2005). Role of the regulatory gene rirA in the transcriptional response of Sinorhizobium meliloti to iron limitation. Appl Environ Microbiol 71, 5969-5982.

Cox, R. J., Hitchman, T. S., Byrom, K. J., Findlow, I. S., Tanner, J. A., Crosby, J. \& Simpson, T. J. (1997). Post-translational modification of heterologously expressed Streptomyces type II polyketide synthase acyl carrier proteins. FEBS Lett 405, 267-272. 
Ditta, G., Schmidhauser, T., Yacobson, E., Lu, P., Liang, X. W., Finlay, D. R. \& Helsinki, D. R. (1985). Plasmids related to the broad host range vector, pRK29, useful for gene cloning and monitoring expression. Plasmid 13, 149-153.

Domínguez-Ferreras, A., Pérez-Arnedo, R., Becker, A., Olivares, J., Soto, M. J. \& Sanjuán, J. (2006). Transcriptome profiling reveals the importance of plasmid pSymB for osmoadaptation of Sinorhizobium meliloti. J Bacteriol 188, 7617-7625.

Dulley, J. R. \& Grieve, P. A. (1975). A simple technique for eliminating interference by detergents in the Lowry method of protein determination. Anal Biochem 64, 136-141.

Epple, G., Van der Drift, K. M. G. M., Thomas-Oates, J. \& Geiger, O. (1998). Characterization of a novel acyl carrier protein, RkpF, encoded by an operon involved in capsular polysaccharide biosynthesis in Sinorhizobium meliloti. J Bacteriol 180, 4950-4954.

Ferguson, G. P., Roop, R. M., II \& Walker, G. C. (2002). Deficiency of Sinorhizobium meliloti bacA mutant in alfalfa symbiosis correlates with the alteration of cell envelope. J Bacteriol 184, 5625-5632.

Figurski, D. H. \& Helsinki, D. R. (1979). Replication of an origincontaining derivative of plasmid RK2 dependent on a plasmid function provided in trans. Proc Natl Acad Sci U S A 76, 16481652.

Finan, T. M., Hartwieg, E., LeMieux, K., Bergman, K., Walker, G. C. \& Signer, E. (1984). General transduction in Rhizobium meliloti. J Bacteriol 159, 120-124.

Flugel, R. S., Hwangbo, Y., Lambalot, R. H., Cronan, J. E. \& Walsh, C. T. (2000). Holo-(acyl carrier protein) synthase and phosphopantetheinyl transfer in Escherichia coli. J Biol Chem 275, 959-978.

Fuqua, C. \& Greenberg, E. P. (2002). Listening in on bacteria: acylhomoserine lactone signalling. Nat Rev Mol Cell Biol 3, 685-695.

Galibert, F., Finan, T. M., Long, S. R., Puhler, A., Abola, P., Ampe, F., Barloy-Hubler, F., Barnett, M. J., Becker, A. \& other authors (2001). The composite genome of the legume symbiont Sinorhizobium meliloti. Science 293, 668-672.

Geiger, O. \& López-Lara, I. M. (2002). Rhizobial acyl carrier proteins and their roles in the formation of bacterial cell surface components that are required for the development of nitrogen-fixing root nodules on legume hosts. FEMS Microbiol Lett 208, 153-162.

Ho, S. N., Hunt, H. D., Horton, R. M., Pullen, J. K. \& Pease, L. (1989). Site directed mutagenesis by overlap extension using polymerase chain reaction. Gene 77, 51-59.

Jackowski, S. \& Rock, C. O. (1983). Ratio of active to inactive forms of acyl carrier protein in Escherichia coli. J Biol Chem 258, 1518615191.

Jiang, Y., Chan, C. H. \& Cronan, J. E. (2006). The soluble acyl-acyl carrier protein synthetase of Vibrio harveyi $\mathrm{B} 392$ is a member of the medium chain acyl-CoA synthetase family. Biochemistry 45, 1000810019.

Kato, J. Y., Funa, N., Watanabe, H., Ohnishi, Y. \& Horinouchi, S. (2007). Biosynthesis of $\gamma$-butyrolactone autoregulators that switch on secondary metabolism and morphological development in Streptomyces. Proc Natl Acad Sci U S A 104, 2378-2383.

Kovach, M. E., Elzer, P. H., Hill, D. S., Robertson, G. T., Farris, M. A., Roop, R. M., II \& Peterson, K. M. (1995). Four new derivatives of the broad-host range vector pBBR1MCS, carrying different antibiotic resistance cassettes. Gene 166, 175-176.
Lai, J. R., Koglin, A. \& Walsh, C. T. (2006). Carrier protein structure and recognition in polyketide and nonribosomal peptide biosynthesis. Biochemistry 45, 14869-14879.

Lambalot, R. H., Gehring, A. M., Flugel, R. S., Zuber, P., LaCelle, M., Marahiel, M. A., Reid, R., Khosla, C. \& Walsh, C. T. (1996). A new enzyme superfamily: the phosphopantetheinyl transferases. Chem Biol 3, 923-936.

López-Lara, I. M. \& Geiger, O. (2000). Expression and purification of four different acyl carrier proteins. Microbiology 146, 839-849.

López-Lara, I. M. \& Geiger, O. (2001). The nodulation protein NodG shows the enzymatic activity of an 3-oxoacyl-acyl carrier protein reductase. Mol Plant Microbe Interact 14, 349-357.

Miller, J. H. (1972). Experiments in Molecular Genetics. Cold Spring Harbor, NY: Cold Spring Harbor Laboratory.

Neuhaus, F. C. \& Baddiley, J. (2003). A continuum of anionic charge: structure, and functions of D-alanyl-teichoic acids in Gram-positive bacteria. Microbiol Mol Biol Rev 67, 686-723.

Olivares, J., Casadesus, J. \& Bedmar, E. J. (1980). Method for testing degree of infectivity of Rhizobium meliloti strains. Appl Environ Microbiol 39, 967-970.

Østeras, M., Boncopagni, E., Vincent, N., Poggi, M. C. \& Le Rudulier, D. (1998). Presence of a gene encoding choline sulfatase in Sinorhizobium meliloti bet operon: choline-O-sulfate is metabolised into glycine betaine. Proc Natl Acad Sci U S A 95, 11394-11399.

Platt, M. W., Miller, K. J., Lane, W. S. \& Kennedy, E. P. (1990). Isolation and characterization of the constitutive acyl carrier protein from Rhizobium meliloti. J Bacteriol 172, 5440-5444.

Post-Beittenmiller, D., Jaworski, J. G. \& Ohlrogge, J. B. (1991). In vivo pools of free and acylated acyl carrier proteins in spinach. Evidence for sites of regulation of fatty acid biosynthesis. J Biol Chem 266, 1858-1865.

Ritsema, T., Gehring, A. M., Stuitje, A. R., van der Drift, K. M., Dandal, I., Lambalot, R. H., Walsh, C. T., Thomas-Oates, J. E., Lugtenberg, B. J. \& Spaink, H. P. (1998). Functional analysis of an interspecies chimera of acyl carrier proteins indicates a specialized domain for protein recognition. Mol Gen Genet 257, 641-648.

Robertsen, B. K., Aitman, P., Darvill, A. G., McNeil, M. \& Alberstein, P. (1981). The structure of acidic extracellular polysaccharides secreted by Rhizobium leguminosarum and Rhizobium trifolii. Plant Physiol 67, 389-400.

Sambrook, J. \& Russell, D. R. (2001). Molecular Cloning: a Laboratory Manual. Cold Spring Harbor, NY: Cold Spring Harbor Laboratory.

Schäfer, A., Tauch, A., Jäger, W., Kalinowski, J., Thierbach, G. \& Pühler, A. (1994). Small mobilizable multi-purpose cloning vectors derived from the Escherichia coli plasmids pK18 and pK19: selection of defined deletions in the chromosome of Corynebacterium glutamicum. Gene 145, 69-73.

Sherwood, M. T. (1970). Improved synthetic medium for the growth of Rhizobium. J Appl Bacteriol 33, 708-713.

Sohlenkamp, C., de Rudder, K. E. \& Geiger, O. (2004). Phosphatidylethanolamine is not essential for growth of Sinorhizobium meliloti on complex culture media. J Bacteriol 186, 1667-1677.

Studier, F. W., Rosenberg, A. H., Dunn, J. J. \& Dubendorff, J. W. (1990). Use of a T7 RNA polymerase to direct expression of cloned genes. Methods Enzymol 185, 60-89.

Edited by: M. F. Hynes 